\title{
Ubiquitin specific protease 22 promotes cell proliferation and tumor growth of epithelial ovarian cancer through synergy with transforming growth factor $\beta 1$
}

\author{
MEI JI, HUIRONG SHI, YA XIE, ZHAO ZHAO, SHUNSHUANG LI, \\ CHENG CHANG, XINGHAN CHENG and YUE LI \\ Department of Obstetrics and Gynecology, The First Affiliated Hospital of Zhengzhou University, \\ Zhengzhou, Henan 450052, P.R. China
}

Received August 21, 2014; Accepted September 22, 2014

DOI: $10.3892 /$ or.2014.3580

\begin{abstract}
Ubiquitin specific protease 22 (USP22) is an oncogene that is upregulated in many cancer types, and aberrant expression of USP22 correlates with clinical outcome. However, its potential functional impact in epithelial ovarian cancer (EOC) has not been determined. Here, we report that USP22 was upregulated in EOC specimens and EOC cell lines with important functional consequences. A high level of USP22 in EOC tissues was associated with advanced clinical FIGO stage, lymph node metastasis and worse prognosis. Patients with higher USP22 expression had shorter relapse-free and overall survival. Depletion of USP22 suppressed cell proliferation in vitro and tumor growth in vivo. We found that inhibition of USP22 suppressed cell proliferation by inducing G1 phase cell cycle arrest through synergy with oncogenic transforming growth factor- $\beta 1$ (TGFB1). Our results indicate that USP22 functions as an oncogene in EOC, and thus USP22 may serve as a potential therapeutic target for individualized EOC treatment.
\end{abstract}

\section{Introduction}

Epithelial ovarian cancer (EOC) is the most lethal gynecological malignancy worldwide $(1,2)$. As the result of advances in surgical management and chemotherapeutic options over the last three decades, the median survival for ovarian cancer patients has improved $(3,4)$. However, progression-free and overall survival have not been significantly altered due to the fact that the origin and pathogenesis of EOC are poorly understood (4). In addition, most patients present with advanced disease, for which effective therapy is currently unavail-

Correspondence to: Dr Huirong Shi, Department of Obstetrics and Gynecology, The First Affiliated Hospital of Zhengzhou University, 1 Jianshe Road, Zhengzhou, Henan 450052, P.R. China

E-mail: huirongshimj@163.com

Key words: USP22, transforming growth factor- $\beta 1$, cell cycle, tumor growth, ovarian carcinoma able (5). Therefore, identification of new molecular targets and therapeutic strategies for EOC patients is urgently needed.

Ubiquitin specific protease 22 (USP22), one of the 11 polycomb/cancer stem cell signature genes that are critical in controlling cell growth and death, is involved in the regulation of pathological processes, including oncogenesis, cell proliferation and cell cycle progression (6-8). Increasing evidence indicates that an oncogenic role of USP22 activation may contribute to tumor progression and predict the prognosis in a variety of human malignancies, including non-small cell lung cancer (9), oral squamous cell carcinoma (10), breast cancer (11), duct (12) and gastric carcinoma (13), and colorectal cancer (14). However, the molecular mechanisms of USP22 in EOC remain to be clarified.

Herein, we evaluated the expression of USP22 in human EOC tissues, and analyzed its correlation with clinicopathological characteristics and the possible prognostic significance. The effects of USP22 on cell proliferation and tumor growth were assessed in vitro as well as in vivo.

\section{Materials and methods}

Patients and follow-up. Paraffin-embedded tissue samples from 86 patients with epithelial ovarian tumors and 30 normal ovaries from hysterectomy specimens resected for non-ovarian disease were obtained from the archives of the Department of Pathology, The First Affiliated Hospital of Zhengzhou University between 2006 and 2012. The tumor cases were histologically confirmed ovarian serous cystadenocarcinoma. The stage of the tumors was assessed according to the International Federation of Gynecology and Obstetrics (FIGO).

The follow-up was completed in 86 patients, and the median follow-up period was 45 months. Follow-up studies included laboratory analysis, physical examination and computed tomography if necessary. Excluding criteria were as follows: i) patients who had undergone chemotherapy or radiotherapy prior to surgery; ii) patients who died within 3 months after surgery; iii) patients whose cause of death remained unknown. The present study was approved by the Ethics Committee of the First Affiliated Hospital of Zhengzhou University. Informed consent was obtained from each participant. 
Cell cultures. Ovarian cancer cell lines SKOV3 and OVCAR3 were purchased from the American Type Culture Collection (ATCC). Cells were cultured in RPMI-1640 supplemented with 10\% FBS (HyClone) and 1\% penicillin streptomycin (Invitrogen) at $37^{\circ} \mathrm{C}$ with $5 \% \mathrm{CO}_{2}$.

Immunohistochemistry. Immunohistochemistry was performed using the avidin-biotin immunoperoxidase technique with an immunohistochemistry kit (ab64261; Abcam) according to the manufacturer's instructions. The primary antibody for the immunohistochemistry was USP22 rabbit polyclonal antibody (1:100; ab4812; Abcam). Paraffinembedded samples were sectioned at a thickness of $4 \mathrm{~mm}$.

RNA extraction and quantitative real-time PCR analysis. Total RNA was extracted using TRIzol reagent. cDNA was synthesized with the PrimeScript RT reagent kit (Takara). Quantitative real-time PCR analysis was carried out to detect mRNA expression using SYBR Premix Ex Taq (Takara), and GAPDH was used as an internal control. The primers for USP22 (115 bp) were 5'-CTA CCA GGA GTC CAC AAA GCAG-3' (forward) and 5'-CAC ATA CGT GGT GAT CTT CCGC-3' (reverse). Primes for oncogenic transforming growth factor- $\beta 1$ (TGFB1) (102 bp) were 5'-CGC GTG CTA ATG GTG GAA A-3' (forward) and 5'-CGC TTC TCG GAG CTC TGA TG-3' (reverse). The primers for GAPDH (138 bp) were 5'-GCA CCG TCA AGG CTG AGA AC-3' (forward) and 5'-TGG TGA AGA CGC CAG TGGA-3' (reverse). All reactions were run in triplicate. The cycle threshold $(\mathrm{Ct})$ values did not differ by $>0.5$ among the triplicates. The USP 22 and TGFB1 levels were normalized to GAPDH to permit calculations of the $2^{-\Delta \Delta C t}$ value.

Lentivirus. USP22 and TGFB1 cDNA was amplified by PCR and subcloned into GV115 vectors (GeneChem, China), designated as pUSP22 and pTGFB1. Lentiviral plasmid vectors encoding short hairpin RNAs (shRNAs) targeting USP22 and TGFB1 were generated by GeneChem (PIEL115080513, PIEL115072998).

Western blotting. Protein lysates were prepared, subjected to SDS-PAGE, transferred onto NC membranes, and blotted according to the standard methods using the USP22 antibody (1:1,000; ab4812; Abcam), TGFB1 antibody (1:1,000; \#8915), cyclin D2 antibody (1:1,000; \#2924) (both from Cell Signaling Technology), anti-Cdk4 antibody (1:1,500; ab137818), anti-Cdk6 antibody (1:1,500; ab151247) and anti-p27kip1 antibody (1:1,500; ab137736) (all from Abcam). The $\beta$-actin antibody (1:1,500; \#4967; Cell Signaling Technology) was used as an internal control.

Cell cycle assay. Cells were transfected with the lentivirus. Nocodazole (100 ng/ml; Sigma-Aldrich) was added $48 \mathrm{~h}$ after transfection, and cells were further incubated for $20 \mathrm{~h}$. Floating and adherent cells were harvested, combined, washed once in phosphate-buffered saline (PBS), and fixed in 70\% ethanol overnight. Staining for DNA content was performed with $50 \mathrm{mg} / \mathrm{ml}$ propidium iodide and $1 \mathrm{mg} / \mathrm{ml}$ RNase A for $30 \mathrm{~min}$. Analysis was performed on a FACScalibur flow cytometer (Becton-Dickinson, Franklin Lakes, NJ, USA) with
Table I. Correlation between USP22 expression and clinicopathologic features of EOC tissues.

\begin{tabular}{|c|c|c|c|c|}
\hline \multirow{2}{*}{$\begin{array}{l}\text { Clinicopathological } \\
\text { features }\end{array}$} & \multirow{2}{*}{$\begin{array}{l}\text { Total } \\
\text { cases }\end{array}$} & \multicolumn{2}{|c|}{$\begin{array}{c}\text { USP22 } \\
\text { expression }\end{array}$} & \multirow[b]{2}{*}{ P-value } \\
\hline & & High & Low & \\
\hline Group & & & & $0.0085^{b}$ \\
\hline Normal tissue & 30 & 19 & 11 & \\
\hline Carcinoma tissue & 86 & 45 & 41 & \\
\hline Age (years) & & & & 0.8828 \\
\hline$\leq 60$ & 37 & 18 & 19 & \\
\hline$>60$ & 49 & 27 & 22 & \\
\hline TNM stage & & & & $0.0028^{b}$ \\
\hline I-II & 28 & 9 & 19 & \\
\hline III-IV & 58 & 36 & 22 & \\
\hline Differentiation & & & & 0.8892 \\
\hline $\mathrm{G} 1$ & 25 & 10 & 15 & \\
\hline $\mathrm{G} 2$ & 23 & 13 & 10 & \\
\hline G3 & 38 & 22 & 16 & \\
\hline Lymph node metastasis & & & & $\mathbf{0 . 0 3 1 3}^{\mathrm{a}}$ \\
\hline Yes & 48 & 31 & 17 & \\
\hline No & 38 & 14 & 24 & \\
\hline Residual tumor size $(\mathrm{cm})$ & & & & 0.0774 \\
\hline$\leq 1$ & 49 & 28 & 21 & \\
\hline$>1$ & 37 & 17 & 20 & \\
\hline
\end{tabular}

aSignificant difference $(\mathrm{P}<0.05)$; ${ }^{\mathrm{b}}$ significant difference $(\mathrm{P}<0.01)$. USP22, ubiquitin specific protease 22.

Cell Quest Pro software. Cell cycle modeling was performed with ModFit 3.0 software (Verity Software House, Topsham, ME, USA).

Cell proliferation assay. The cell proliferation assay was performed with WST-8 Cell Counting Kit-8 (Beyotime, Jiangsu, China) according to the manufacturer's instructions. Transfected cells were seeded in 96-well plates and cultured overnight. CCK-8 solution $(10 \mu \mathrm{l})$ was added to 96 -well plates, and the cultures were incubated for $2 \mathrm{~h}$ at $37^{\circ} \mathrm{C}$. Cell proliferation was documented every $24 \mathrm{~h}$ by measuring the absorbance at $450 \mathrm{~nm}$ in an automatic microplate reader (Bio-Rad, Hercules, CA, USA). The results presented are averages from 3 independent experiments.

In vivo tumor growth assay. Female athymic BALB/c nude mice were purchased from Vital River, a Charles River Company (Beijing, China) and were maintained in specific pathogen-free conditions. Animal care and experimental protocols were conducted in accordance with the guidelines of Zhengzhou University Medical Experimental Animal Care Commission. OVCAR3 cells $\left(5 \times 10^{7}\right)$ infected with the lentivirus encoding shUSP22 [at a multiplicity of infection (MOI) of 50] were subcutaneously injected into the flank of the nude mice (6 in each group, female BALB/c nu/nu, 4-5 weeks), and 


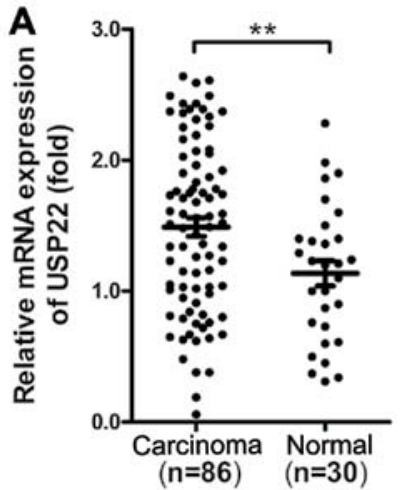

D

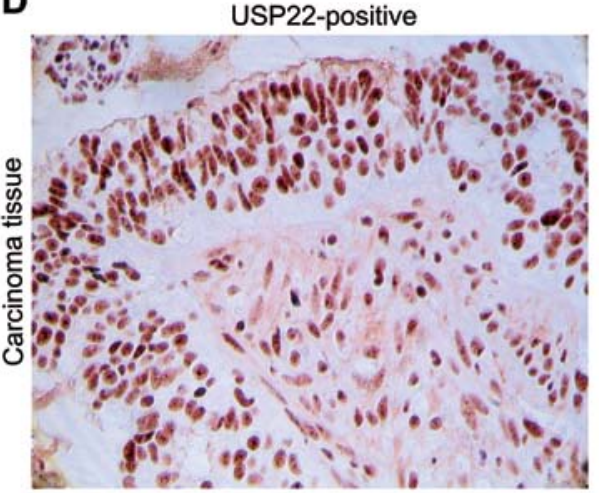

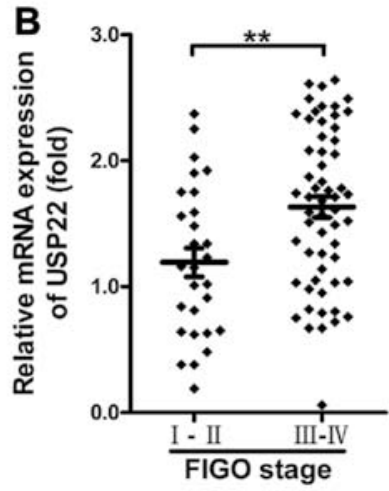

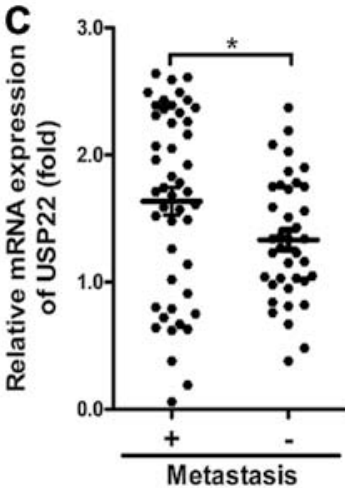

E

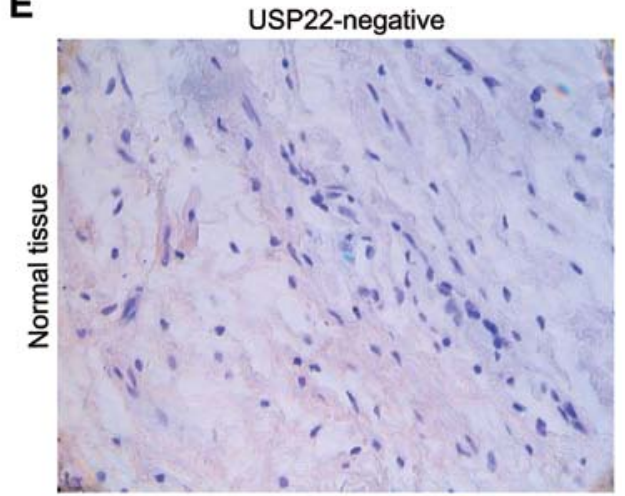

Figure 1. USP22 is upregulated in human EOC tissues. (A) The expression of USP22 was determined by real-time qPCR in human epithelial ovarian cancer tissues $(n=86)$ and normal ovarian tissues $(n=30)$. The median value of all 86 cases was chosen as the cut-off point for separating the USP22-high expression and USP22-low expression groups. (B) Clinical FIGO stage and (C) lymph node metastasis were positively correlated with USP22 expression in the human epithelial ovarian cancer tissues. The central horizontal line represents the mean value. The absence $(n=38)$ or presence $(n=48)$ of lymph node metastasis is indicated as '-' and '+', respectively. "P<0.05, ${ }^{* *} \mathrm{P}<0.01$. (D and E) USP22 expression was also detected by immunohistochemical staining in cancer and normal tissues. Magnification, $\mathrm{x} 200$. USP22, ubiquitin specific protease 22; EOC, epithelial ovarian cancer.

OVCAR3 cells treated with the lentivirus encoding shNon were used as negative control (NC). Tumor formation in nude mice was monitored over a 6 -week period. Tumor volume (V) was measured twice weekly using a caliper and calculated as $\mathrm{V}=\left(\right.$ tumor length $\mathrm{x}$ width $\left.{ }^{2}\right) / 2$.

Statistical analysis. Overall survival (OS) was defined as the interval between surgery and death or between surgery and the last observation time point. For surviving patients, the data were censored at the last follow-up. Relapse-free survival (RFS) was defined as the interval between the date of surgery and the date of diagnosis of first recurrence. Kaplan-Meier plots and the Cox proportional hazard regression analysis, which were applied to identify the prognostic factors, were performed with SPSS version 13.0 (SPSS, Inc., Chicago, IL, USA). Associations between the OS and the molecular changes or clinical characteristics were analyzed initially by a univariate Cox proportional hazards regression analysis. Significant prognostic factors found in the univariate analysis were evaluated further by multivariate Cox regression analysis.

The data are expressed as the means \pm standard error of the mean (SEM) from at least 3 independent experiments. The differences between groups were analyzed by Student's t-test when two groups were compared or by one-way ANOVA when more than two groups were compared. Analyses were performed with GraphPad Prism version 5 (GraphPad
Software, Inc., San Diego, CA, USA). All statistical tests were two-sided and $\mathrm{P}<0.05$ was considered to indicate a statistically significant result.

\section{Results}

USP22 is overexpressed in primary EOC and is involved in EOC development. We examined the expression levels of USP22 in 86 human EOC tissues and 30 normal ovarian tissues by quantitative real-time PCR analysis, as well as immunohistochemistry. Our data showed that USP22 expression in the EOC tissues was overexpressed in comparison with that in the normal ovarian tissues $(\mathrm{P}=0.0085$; Fig. $1 \mathrm{~A}, \mathrm{D}$ and $\mathrm{E})$. We further analyzed the association of USP22 expression with clinicopathological parameters in EOC. As shown in Table I, the USP22 expression in EOC tissues was significantly correlated with advanced clinical FIGO stage $(\mathrm{P}=0.0028$; Table I, Fig. 1B) and lymph node metastasis $(\mathrm{P}=0.0313$; Table I, Fig. 1C). However, no significant associations were found between USP22 expression and patient age, histological grade or tumor size $(\mathrm{P}>0.05$; Table I). These results suggest that a higher level of USP22 expression may be involved in EOC progression.

High levels of USP22 expression are associated with worse prognosis in EOC patients. The potential association between 
Table II. Univariate survival analysis of RFS and OS in patients with epithelial ovarian cancer.

\begin{tabular}{|c|c|c|c|c|c|c|c|}
\hline \multirow[b]{2}{*}{ Variables } & \multirow[b]{2}{*}{ Cases } & \multicolumn{2}{|c|}{ RFS (months) } & \multirow[b]{2}{*}{ P-value } & \multicolumn{2}{|c|}{ OS (months) } & \multirow[b]{2}{*}{ P-value } \\
\hline & & Mean & Median & & Mean & Median & \\
\hline \multicolumn{8}{|c|}{ Expression group } \\
\hline USP22 low & 41 & 37.218 & 44 & \multirow[t]{2}{*}{$0.030^{\mathrm{a}}$} & 45.742 & 52 & \multirow[t]{2}{*}{$0.027^{\mathrm{a}}$} \\
\hline USP22 high & 45 & 27.212 & 24 & & 38.643 & 36 & \\
\hline \multicolumn{8}{|l|}{ Age (years) } \\
\hline$\leq 60$ & 37 & 34.481 & 24 & \multirow[t]{2}{*}{0.403} & 42.625 & 44 & \multirow[t]{2}{*}{0.684} \\
\hline$>60$ & 49 & 31.992 & 28 & & 41.876 & 36 & \\
\hline \multicolumn{8}{|l|}{ TNM stage } \\
\hline I-II & 28 & 41.107 & - & \multirow[t]{2}{*}{$0^{0.008}{ }^{b}$} & 46.282 & - & \multirow[t]{2}{*}{$0.011^{a}$} \\
\hline III-IV & 58 & 29.453 & 24 & & 40.637 & 36 & \\
\hline \multicolumn{8}{|l|}{ Differentiation } \\
\hline G1 & 25 & 37.189 & 48 & \multirow[t]{3}{*}{0.393} & 44.284 & 52 & \multirow[t]{3}{*}{0.585} \\
\hline $\mathrm{G} 2$ & 23 & 33.853 & 32 & & 42.383 & 52 & \\
\hline G3 & 38 & 28.616 & 24 & & 40.566 & 36 & \\
\hline \multicolumn{8}{|c|}{ Lymph node metastasis } \\
\hline Yes & 48 & 27.738 & 18 & \multirow[t]{2}{*}{$0.017^{a}$} & 38.914 & 36 & \multirow[t]{2}{*}{$0.049^{a}$} \\
\hline No & 38 & 38.980 & 48 & & 46.440 & 48 & \\
\hline \multicolumn{8}{|c|}{ Residual tumor size (cm) } \\
\hline$\leq 1$ & 49 & 37.442 & 44 & \multirow[t]{2}{*}{0.055} & 43.980 & 40 & \multirow[t]{2}{*}{$0.045^{a}$} \\
\hline$>1$ & 37 & 29.516 & 24 & & 40.243 & 36 & \\
\hline
\end{tabular}

${ }^{\text {aS }}$ ignificant difference $(\mathrm{P}<0.05)$; ${ }^{\mathrm{b}}$ significant difference $(\mathrm{P}<0.01)$. RFS, relapse-free survival; OS, overall survival; USP22, ubiquitin specific protease 22 .
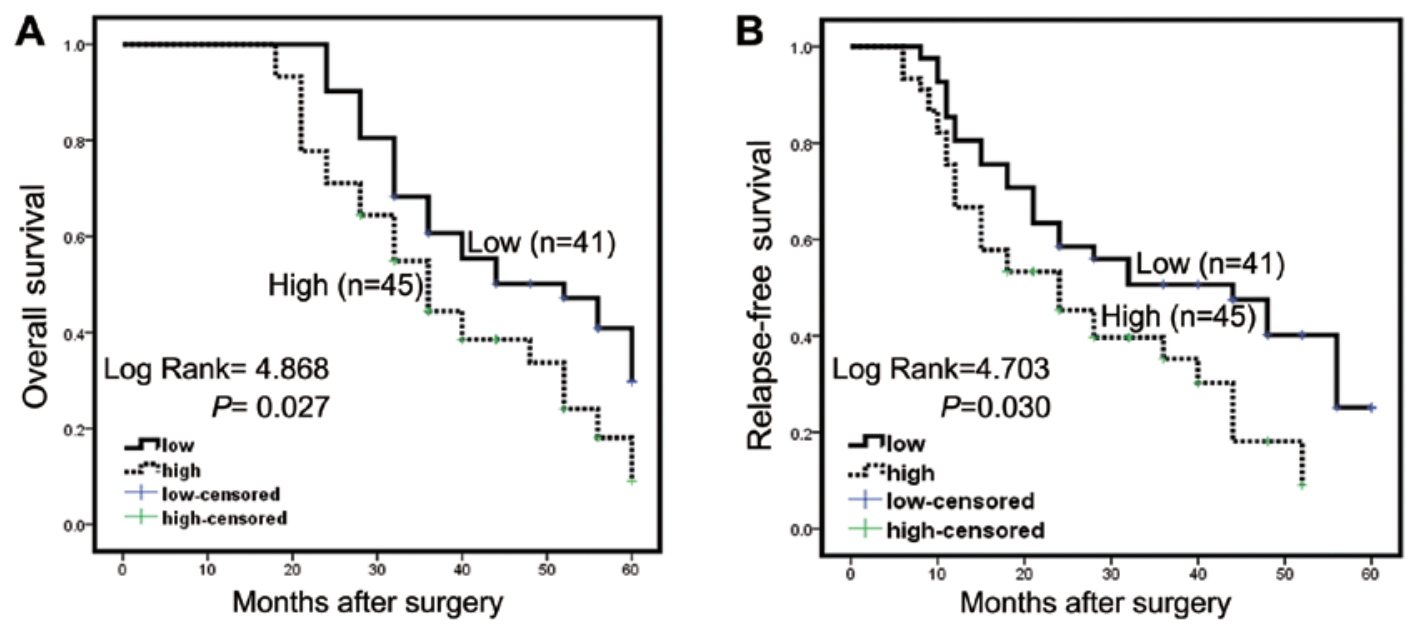

Figure 2. High level of USP22 expression in EOC tissues is associated with worse prognosis. (A) Overall survival. (B) Relapse-free survival. USP22, ubiquitin specific protease 22; EOC, epithelial ovarian cancer.

the USP22 expression level and RFS or OS was evaluated. Kaplan-Meier analysis plots revealed that patients with higher USP22 levels $(n=45)$ had a mean OS of 38.6 months, whereas patients with lower USP22 levels $(n=41)$ had a mean OS of 45.7 months. ( $\mathrm{P}=0.027$; Fig. $2 \mathrm{~A}$, Table II). We also found that patients with higher USP22 levels had a mean RFS of 27.2 months, whereas patients with lower USP22 levels had a mean
RFS of 37.2 months ( $\mathrm{P}=0.030$; Fig. 2B, Table II). However, multivariate Cox regression analysis showed that USP22 was not an independent risk factor for RFS and OS.

Inhibition of USP22 suppresses the proliferation and tumorigenesis of ovarian cancer cells in vitro and in vivo. We transfected the ovarian cancer cell lines SKOV3 and OVCAR3 
A

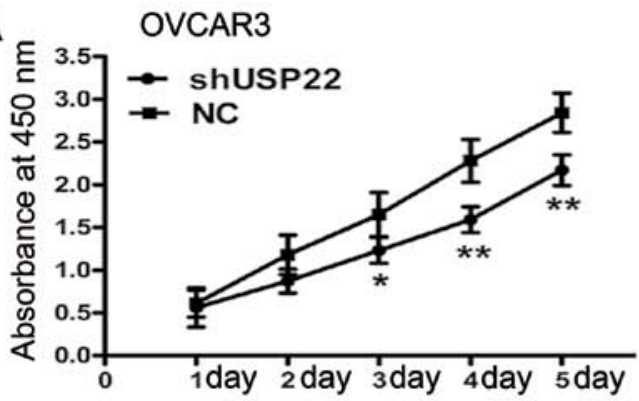

C
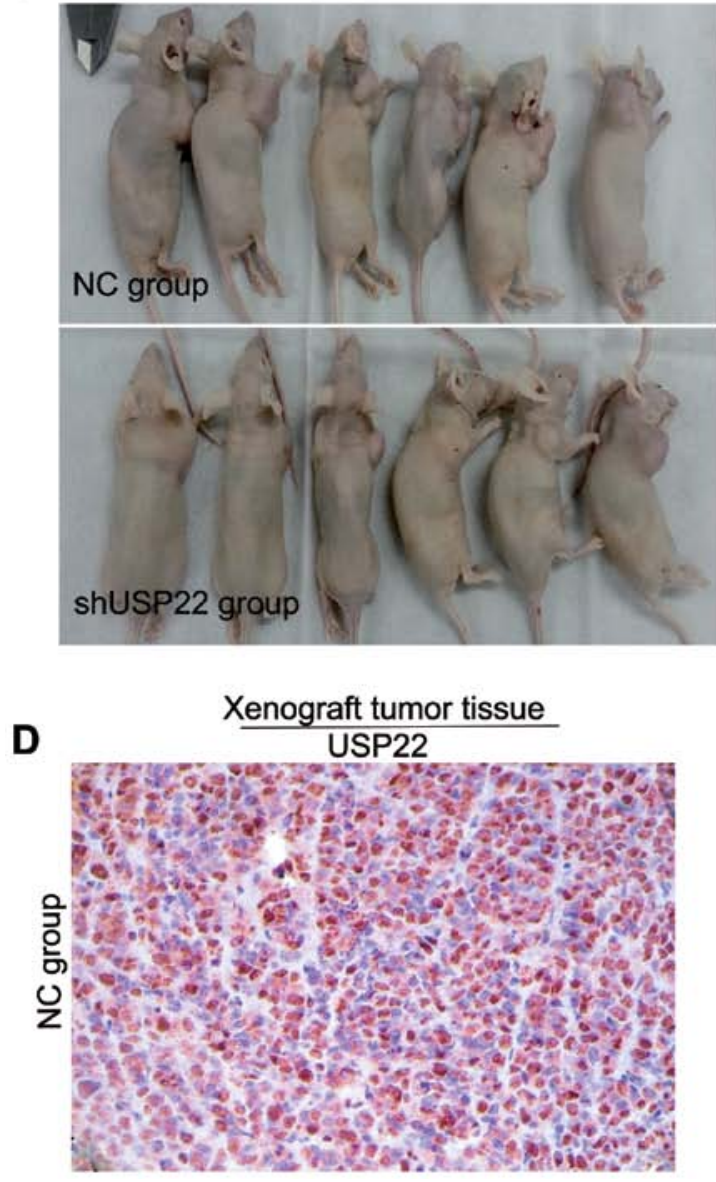

B

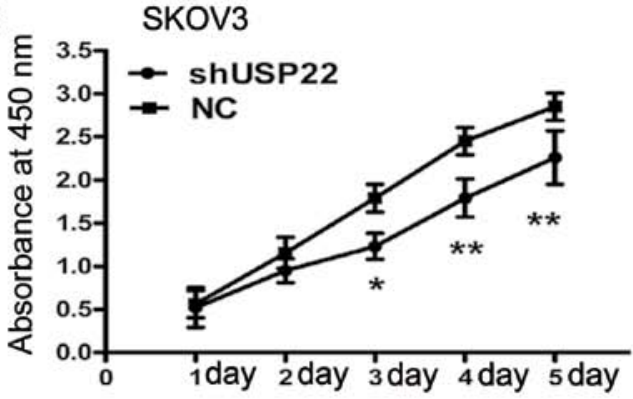

Tumor growth in nude mice
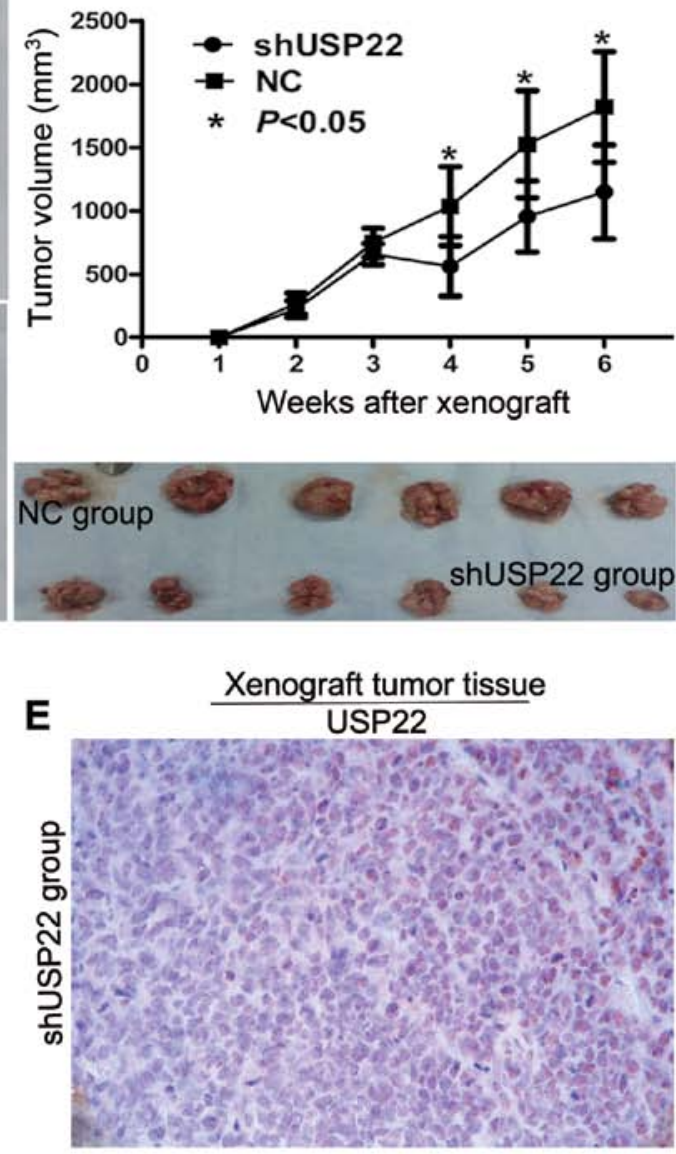

Figure 3. Depletion of Usp22 suppresses tumor growth in vitro and in vivo. (A and B) OVCAR3 and SKOV3 cells were transfected with lentivirus expression shRNA molecules directed against USP22 or the empty vector. Forty-eight hours after transfection, the CCK-8 assay was used to measure cell growth abilities. Negative control (NC) cells transfected with the empty vector. (C) Xenograft tumor growth was monitored at the indicated weeks after tumor implantation. Tumors developed in the implanted sites in all the 12 mice. The dissected tumors were measured for length (L) and width (W) with calipers and the tumor volumes (V) were calculated according to the formula: $\mathrm{V}=\left(\mathrm{L} \mathrm{x} \mathrm{W}^{2}\right) \times 0.5$. ${ }^{*} \mathrm{P}<0.05 ;{ }^{* *} \mathrm{P}<0.01$. (D and E) Representative images of USP22 immunostaining in OVCAR3 xenograft tumors. Magnification, x200. USP22, ubiquitin specific protease 22.

with the USP22 lentivirus encoding shRNA to knock down USP22, and examined the effects on cellular proliferation. CCK-8 assays revealed that depletion of USP22 significantly decreased the growth rate of both ovarian cancer cell lines, compared to the negative control transfected empty vector cells (Fig. 3A and B).

The effect of USP22 on cell growth was further confirmed by in vivo assay in xenografts. As shown in Fig. 3C, the tumors in the OVCAR3/NC (empty vector control) group grew more rapidly than the tumors in the USP22-deficient OVCAR3/ shUSP22 group. Significant differences in average tumor size were observed on day 28 and at the end of the observation post injection (tumor volume on day 35, $0.955 \mathrm{vs} .1 .526 \mathrm{~mm}^{3}$, $\mathrm{P}=0.021$; tumor volume on day $42,1.149 \mathrm{vs} .1 .820 \mathrm{~mm}^{3}$, $\mathrm{P}=0.017$; Fig. 3C). Immunohistochemistry staining confirmed that the tumors of the USP22-deficient OVCAR3/shUSP22 group displayed much lower USP22 levels than the tumors from the NC control group (Fig. 3D and E).

Collectively, both the in vitro and in vivo studies suggest that USP22 promotes EOC progression by promoting cellular proliferation and tumor growth.

Inhibition of USP22 suppresses ovarian cell proliferation by inducing 61 phase cell cycle arrest. USP22 is known to play 
A

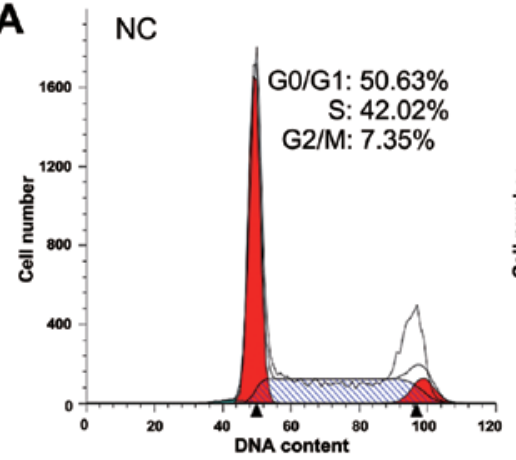

C

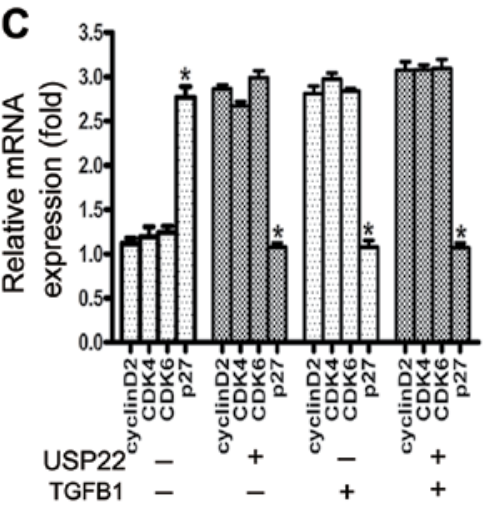

shUSP22

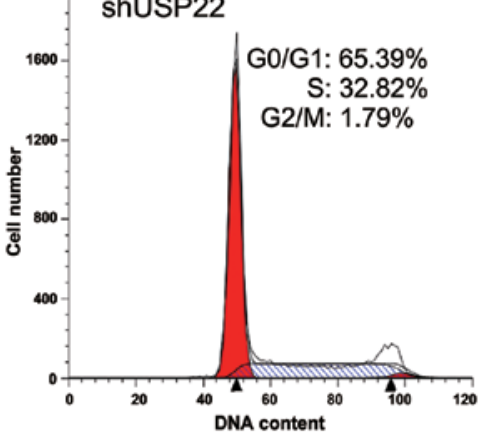

D

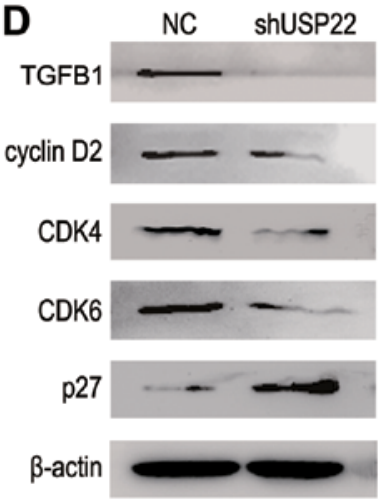

B

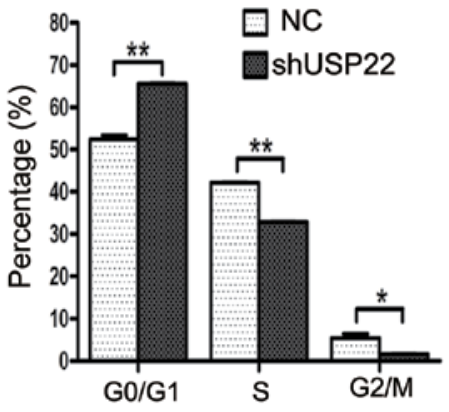

E

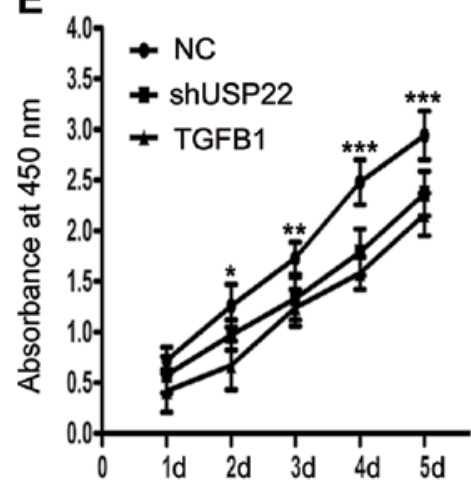

Figure 4. Depletion of USP22 suppresses tumor cell proliferation by inducing G1 phase cell cycle arrest through synergy with the oncogene TGFB1. (A and B) OVCAR3 cells were transfected with lentivirus expression shRNA molecules directed against USP22 or transfected with empty vector cells and were stained with propidium iodide. Cell cycle profile was determined by flow cytometry. (C) The effects of USP22 depletion were consistent with those of TGFB1 depletion. OVCAR3 cells that were co-tranfected with the following lentivirus combinations NC (empty vector), USP22/NC, NC/TGFB1, USP22/TGFB1 were used to detect TGFB1 target gene expression by real-time qPCR. GAPDH was used as an internal control. (D) Western blot assays show the expression of TGFB1 and its target genes in OVCAR3 cells from $\mathrm{C}$. $\beta$-actin was used as an internal immunoblotting control. (E) CCK-8 assays show the cell growth of OVCAR3 cells depleted of USP22 or TGFB1 by shRNA interference. Negative control (NC) cells transfected with the empty vector. "P<0.05; ${ }^{* *} \mathrm{P}<0.01,{ }^{* * *} \mathrm{P}<0.001$. USP22, ubiquitin specific protease 22; TGFB1, oncogenic transforming growth factor- $\beta 1$.

a crucial role in cell cycle regulation (8). We further verified the cell cycle distribution of the USP22-deficient OVCAR3 cells by flow cytometry, to explore the possible mechanism by which USP22 regulates ovarian cancer cell proliferation. Flow cytometric analysis of cells transfected with USP22 shRNA showed a consistent significant decrease in the percentage of cells in the $S$ phase and an increase in the G1 phase compared with the negative control cells (Fig. 4A and B). These results indicate that depletion of USP22 results in G1 phase cell cycle arrest in ovarian cancer cells.

TGFB1 is known as a multifunctional growth factor, which participates in the regulation of cell proliferation, differentiation, cell cycle arrest and other functions in many cell types (15-17). Many cells have TGFB1 receptors (18), and TGFB1 is frequently upregulated in ovarian cancer cells $(19,20)$. Thus, we hypothesized that USP22 may synergize TGFB1 to promote tumor cell growth via regulating TGFB1 downstream cell cycle signaling pathways. We determined the mRNA levels of several TGFB1 target genes that are involved in cell cycle signaling in OVCAR3 cells by qPCR and western blotting including cyclin D2, cyclin-dependent kinase 4 and 6 (CDK4 and CDK6), and cell cycle inhibitor p27kip1. We found that the levels of cyclin D2, CDK4, CDK6 were decreased by $>1.5$-fold, whereas the levels of tumor suppressor p27kip1 were increased at least 2-fold after depletion of USP22 or TGFB1 expression (Fig. 4C and D). We also found that the protein levels of TGFB1 were decreased after depletion of USP22 expression (Fig. 4D). In addition, CCK-8 assays indicated that depletion of TGFB1 significantly reduced the growth rate of OVCAR3 cells (Fig. 4E). These results suggest that USP22 may accelerate ovarian cancer cell cycle progression via synergizing with TGFB1 to regulate the TGFB1 downstream cell cycle pathway, which in turn, promotes ovarian cancer cell proliferation.

\section{Discussion}

Deubiquitination is an important process for numerous cellular mechanisms $(21,22)$, and the imbalance of this process can cause severe diseases including cancer $(23,24)$. USP22, a novel deubiquitinating enzyme, is involved in the regulation of numerous pathological processes. Based on in vitro and in vivo evidence, we propose that USP22 is capable of suppressing EOC cell proliferation and tumor growth, and overexpression of USP22 may facilitate EOC progression.

Recently, increasing experimental and clinical observations reveal that USP22 plays a crucial role in tumor progression (25) and influences clinical prognosis of several human malignancies (26). In human invasive breast cancer, elevated expression of USP22 was found to be positively related to lymph node metastasis and patient recurrence (11). In human non-small cell lung cancer, positive expression of 
USP22 is significantly correlated to tumor stage and poor overall survival (9). In human colorectal cancer, increased mRNA expression of USP22 was associated with advanced tumor stage and the high likelihood of therapy failure after radical resection (27). In esophageal squamous cell carcinoma, USP22 is an independent prognosticator for unfavorable disease-specific survival (28). In oral squamous cell carcinoma, USP22 is associated with recurrence and prognosis (10). However, to our knowledge, the detailed mechanism surrounding any role that USP22 may play in EOC development has not been reported previously.

In the present study, we identified that the expression level of USP22 was significantly overexpressed in EOC tissues compared to normal ovarian tissues. Moreover, increased USP22 expression was associated with advanced clinical FIGO stage and lymph node metastasis. These findings strongly suggest that USP22 activation may play an oncogenic role in promoting tumor progression of EOC. To further validate the potential clinical utility of USP22, we evaluated the prognostic value of USP22 in EOC patients. Univariate analysis revealed that cancer patients with USP22 overexpression had a significantly worse RFS and OS after radical surgery, compared with the patients in the low expression group. However, multivariate analysis showed that the expression level of USP22 was not an independent prognostic factor in EOC patients. The lack of large-scale clinical samples may have influenced the results. Further studies are needed to expand the large-scale study to evaluate the prognostic impact of USP22 expression in ovarian cancer patients.

Previous studies have demonstrated that USP22 is involved in the regulation of tumor growth and cell cycle progression. USP22 is a dedicated subunit of the hSAGA complex, and is required for the transcription of target genes regulated by the c-Myc oncoprotein (8). c-Myc is a downstream target gene of the TGFB1 signaling pathway. TGFB1 encodes the transforming growth factor $\beta$ family of cytokines, which are multifunctional peptides that regulate proliferation, differentiation, migration, cell cycle and other functions in many cell types (16-18). Many cells have TGFB1 receptors, and the protein positively and negatively regulates many other growth factors (29-31). We found that depletion of USP22 could inhibit cell growth, induce G1 cell cycle arrest in ovarian cancer cells, and suppress tumorigenesis in a nude mouse model of EOC xenografts. We observed a weak but significant correlation between high TGFB1 and high USP22 expression in tumor cells. We also examined the expression of a panel of cell cycle regulators on TGFB1 downstream pathways. We found that the expression of cyclin D2, CDK4, CDK6 and p27kip1 was indirectly regulated by USP22 dependent on TGFB1. Depletion of TGFB1 significantly reduced the growth rate of ovarian cancer cells indicating that a similar connection may be present in the cancer cells. USP22 may stimulate cell proliferation and cell cycle through TGFB1 release from tumor cells. Supporting our findings, USP22 was also found to influence the cell cycle of human colorectal cancer cell line HCT116a by downregulation of MUP expression (14). In human bladder cancer cell line EJ (32), knockdown of USP22 expression by siRNA downregulated the expression of Mdm2 and cyclin E, resulting in the upregulated expression of p53 and p21 leading to cell cycle arrest and inhibition of cell proliferation.
The present study, together with the findinds from other groups, has revealed a mechanism involving the regulation of cell proliferation and tumor growth by USP22 through synergy with the oncoprotein TGFB1. Further study is warranted to confirm that this novel deubiquitinating enzyme has clinical implication as an individualized treatment strategy for EOC patients.

\section{Acknowledgements}

This study was supported by the National Natural Science Foundation of China (grant no. 81202070), and the Basic and Advanced Technology Research Foundation from the Science and Technology Department of Henan Province (grant no. 112102310105)

\section{References}

1. Siegel R, Naishadham D and Jemal A: Cancer statistics, 2013. CA Cancer J Clin 63: 11-30, 2013.

2. Jemal A, Bray F, Center MM, Ferlay J, Ward E and Forman D: Global cancer statistics. CA Cancer J Clin 61: 69-90, 2011.

3. Trimbos JB, Vergote I, Bolis G, et al: Impact of adjuvant chemotherapy and surgical staging in early-stage ovarian carcinoma: European Organisation for Research and Treatment of CancerAdjuvant ChemoTherapy in Ovarian Neoplasm trial. J Natl Cancer Inst 95: 113-125, 2003.

4. Kurman RJ and Shih IeM: Molecular pathogenesis and extraovarian origin of epithelial ovarian cancer - shifting the paradigm. Hum Pathol 42: 918-931, 2011.

5. Hess LM, Rong N, Monahan PO, Gupta P, Thomaskutty C and Matei D: Continued chemotherapy after complete response to primary therapy among women with advanced ovarian cancer: a meta-analysis. Cancer 116: 5251-5260, 2010.

6. Lin Z, Yang H, Kong Q, et al: USP22 antagonizes p53 transcriptional activation by deubiquitinating Sirt1 to suppress cell apoptosis and is required for mouse embryonic development. Mol Cell 46: 484-494, 2012.

7. Liu YL, Jiang SX, Yang YM, Xu H, Liu JL and Wang XS: USP22 acts as an oncogene by the activation of BMI-1-mediated INK4a/ARF pathway and Akt pathway. Cell Biochem Biophys 62 : 229-235, 2012

8. Zhang XY, Varthi M, Sykes SM, et al: The putative cancer stem cell marker USP22 is a subunit of the human SAGA complex required for activated transcription and cell-cycle progression. Mol Cell 29: 102-111, 2008

9. Hu J, Liu YL, Piao SL, Yang DD, Yang YM and Cai L: Expression patterns of USP22 and potential targets BMI-1, PTEN, p-AKT in non-small-cell lung cancer. Lung Cancer 77: 593-599, 2012.

10. Piao S, Liu Y, Hu J, et al: USP22 is useful as a novel molecular marker for predicting disease progression and patient prognosis of oral squamous cell carcinoma. PLoS One 7: e42540, 2012.

11. Zhang Y, Yao L, Zhang X, et al: Elevated expression of USP22 in correlation with poor prognosis in patients with invasive breast cancer. J Cancer Res Clin Oncol 137: 1245-1253, 2011.

12. Piao S, Ma J, Wang W, et al: Increased expression of USP22 is associated with disease progression and patient prognosis of salivary duct carcinoma. Oral Oncol 49: 796-801, 2013.

13. Yang DD, Cui BB, Sun LY, et al: The co-expression of USP22 and BMI-1 may promote cancer progression and predict therapy failure in gastric carcinoma. Cell Biochem Biophys 61: 703-710, 2011.

14. Xu H, Liu YL, Yang YM and Dong XS: Knock-down of ubiquitin-specific protease 22 by micro-RNA interference inhibits colorectal cancer growth. Int J Colorectal Dis 27: 21-30, 2012.

15. Tian $M$ and Schiemann WP: The TGF- $\beta$ paradox in human cancer: an update. Future Oncol 5: 259-271, 2009.

16. Bierie B and Moses HL: Tumour microenvironment: TGF $\beta$ : the molecular Jekyll and Hyde of cancer. Nat Rev Cancer 6: 506-520, 2006.

17. Wenner CE and Yan S: Biphasic role of TGF- $\beta 1$ in signal transduction and crosstalk. J Cell Physiol 196: 42-50, 2003. 
18. Principe DR, Doll JA, Bauer J, et al: TGF- $\beta$ : duality of function between tumor prevention and carcinogenesis. J Natl Cancer Inst 106: djt369, 2014

19. Cho MS, Bottsford-Miller J, Vasquez HG, et al: Platelets increase the proliferation of ovarian cancer cells. Blood 120: 4869-4872, 2012.

20. Cai J, Tang H, Xu L, et al: Fibroblasts in omentum activated by tumor cells promote ovarian cancer growth, adhesion and invasiveness. Carcinogenesis 33: 20-29, 2012.

21. Yang Y, Kitagaki J, Wang H, Hou DX and Perantoni AO: Targeting the ubiquitin-proteasome system for cancer therapy. Cancer Sci 100: 24-28, 2009.

22. Song L and Rape M: Reverse the curse - the role of deubiquitination in cell cycle control. Curr Opin Cell Biol 20: 156-163, 2008.

23. Shen M, Schmitt S, Buac D and Dou QP: Targeting the ubiquitin-proteasome system for cancer therapy. Expert Opin Ther Targets 17: 1091-1108, 2013.

24. Hussain S, Zhang Y and Galardy PJ: DUBs and cancer: the role of deubiquitinating enzymes as oncogenes, non-oncogenes and tumor suppressors. Cell Cycle 8: 1688-1697, 2009.

25. Kapoor S: Usp22 and its evolving role in systemic carcinogenesis. Lung Cancer 79: 191, 2013.

26. Schrecengost RS, Dean JL, Goodwin JF, et al: USP22 regulates oncogenic signaling pathways to drive lethal cancer progression. Cancer Res 74: 272-286, 2014.
27. Liu YL, Yang YM, Xu H and Dong XS: Increased expression of ubiquitin-specific protease 22 can promote cancer progression and predict therapy failure in human colorectal cancer. J Gastroenterol Hepatol 25: 1800-1805, 2010.

28. Li J, Wang Z and $\mathrm{Li} \mathrm{Y}$ : USP22 nuclear expression is significantly associated with progression and unfavorable clinical outcome in human esophageal squamous cell carcinoma. J Cancer Res Clin Oncol 138: 1291-1297, 2012.

29. Kajdaniuk D, Marek B, Borgiel-Marek H and Kos-Kudla B: Transforming growth factor $\beta 1$ (TGF $\beta 1$ ) in physiology and pathology. Endokrynol Pol 64: 384-396, 2013.

30. Moses $\mathrm{H}$ and Barcellos-Hoff MH: TGF- $\beta$ biology in mammary development and breast cancer. Cold Spring Harb Perspect Biol 3: a003277, 2011.

31. Anscher MS: Targeting the TGF- $\beta 1$ pathway to prevent normal tissue injury after cancer therapy. Oncologist 15: 350-359, 2010.

32. Lv L, Xiao XY, Gu ZH, Zeng FQ, Huang LQ and Jiang GS: Silencing USP22 by asymmetric structure of interfering RNA inhibits proliferation and induces cell cycle arrest in bladder cancer cells. Mol Cell Biochem 346: 11-21, 2011 